\title{
Rheology of Suspensions of Hydrophilic and Hydrophobic Solid Particles in Nonaqueous Media
}

\author{
A. J. G. VAN DIEMEN, F. W. A. M. SCHREUDER, AND H. N. STEIN
}

Laboratory of Colloid Chemistry, Eindhoven University of Technology, Eindhoven, The Netherlands

Received October 26, 1983; accepted May 10, 1984

\begin{abstract}
Coagulation of suspensions of hydrophobic particles becomes apparent if the hydrophilic/lipophilic balance (HLB) value of the medium is larger than 14; suspensions of hydrophilic solid particles show no distinct tendency toward coagulation in the media investigated (HLB $\geqslant 4.5$ ). As a criterion for coagulation, rheological, and sedimentation characteristics are employed. The rheological behavior of these suspensions indicates that both in the Newtonian and in the non-Newtonian range, the energy dissipation during stationary flow is proportional to the viscosity of the suspension medium. This excludes any significant contribution to this energy dissipation from the increase and decrease of potential energy between the suspended particles (breaking and renewed formation of interparticle bonds). 1985 Academic Press, Inc.
\end{abstract}

\section{INTRODUCTION}

The present study is concerned with the question: which characteristics of a suspension medium are required for coagulation to become apparent? Previous investigations (1, 2) had shown that suspensions of hydrophilic and of hydrophobic particles may behave differently if the polarity of the suspension medium favors coagulation of one type. Thus, suspensions of hydrophilic glass particles in glycerol did not show any sign of coagulation, whereas in suspensions of hydrophobic particles (glass treated by dimethyldichlorosilane) coagulation was very pronounced (2). However, in di-2-ethylhexyl-phthalate as a medium no difference in rheological properties was found between suspensions of hydrophilic and hydrophobic particles; this was considered as evidence for absence of coagulation in both types of suspension.

For the sake of brevity, untreated glass will be designated in the present paper as "hydrophilic," and glass treated by dimethyldichlorosilane as "hydrophobic." Differences between glasses made hydrophobic by different techniques may occur. However, electron micrographs of glass treated by dimethyldichlorosilane (2) showed a coherent polymer layer on the glass; thus we are convinced that our "hydrophobic" particles are at or near the one extreme of the scale.

The previous investigations were restricted either to liquids of rather complex structures (polymers) (1) or to simple liquids of extreme polarity characteristics (2): glycerol (which forms homogeneous mixtures with water in all proportions) on the one hand, and di-2ethylhexyl phthalate (solubility in water $<0.01$ mass\% (3)) on the other. In order to fix the characteristics necessary for coagulation of hydrophobic particles more precisely, extension of the earlier experiments to a medium with intermediate character was required. Moreover, a check on the absence of coagulation of hydrophilic particles, which was inferred in the previous investigations from rheology only, by sedimentation experiments appeared desirable.

In order to express the hydrophilic or hydrophobic character of a suspension medium in a number, recourse will be made in the present paper to the HLB value (4) which is that percentage of the formula mass which 
can be considered as "hydrophilic," divided by 5 . Thus, glycerol should be awarded a HLB value of $20(100 \%$ of the mass of a molecule being hydrophilic), whereas di-2ethylhexyl phthalate has a HLB value of 4.5 (22.5\% of the mass of a molecule preferring the aqueous phase at an oil/water interface).

The present investigation bridges the gap between these extremes, by studying suspensions of both hydrophilic and hydrophobic glass particles in the bis-methyl glycol ester of phthalic acid, having a HLB value of 14.6.

\section{EXPERIMENTAL}

\section{Materials}

Glass Ballotini: from Tamson (Zoetermeer); density $2.75 \times 10^{3} \mathrm{~kg} \mathrm{~m}^{-3}$. The fraction $36<d<50 \mu \mathrm{m}$ ( $d=$ diameter) was isolated by dry sieving.

The particles were spherical (for a SEM, see Ref. (2)). Part of them was made hydrophobic by the method of Kao et al. $(1,2)$. Typical size distributions, determined in a Micrometrics Sedigraph with di-2-ethylhexyl phthalate as a medium, are shown in Table I.

Di-2-ethylhexyl phthalate (dioctyl phthalate = DOP): ex Hoechst ("technical purity"); viscosity $\left(20^{\circ} \mathrm{C}\right) 80.31 \times 10^{-3} \mathrm{~Pa} \mathrm{sec}$.

Phthalic acid bis-methyl glycol ester (di(methoxyglycol) phthalate, = DMGP): $e x$ Fluka "chemical purity"); viscosity $\left(20^{\circ} \mathrm{C}\right) 29,12$ $\times 10^{-3} \mathrm{~Pa} \mathrm{sec}$; density $1.166 \times 10^{3} \mathrm{~kg} \mathrm{~m}^{-3}$.

Glycerol 87\%: ex Merck ("zur Analyse"); viscosity $\left(20^{\circ} \mathrm{C}\right) 149.13 \times 10^{-3} \mathrm{~Pa} \mathrm{sec}$.

\section{Apparatus}

Epprecht rotational viscometer type Rheomat 15T-FC (Contraves, A. G., Zürich).

TABLE I

Hydrodynamic Diameter of Glass Ballotini ( $\mu \mathrm{m})$

\begin{tabular}{ccc}
\hline Mass $\%$ & Hydrophilic & Hydrophobic \\
\hline 20 & $<37$ & $<38.5$ \\
50 & $<43$ & $<44$ \\
80 & $<46$ & $<47.5$ \\
\hline
\end{tabular}

Measuring systems B and C were employed, with shear rates varying between 3.13 and $197.1 \mathrm{sec}^{-1}$, or 2.18 and $137.1 \mathrm{sec}^{-1}$, respectively.

\section{Procedures}

Preparation of pastes. Pastes were prepared by stirring by hand and afterward introduced into the rheometer. The latter operation presented problems at solid volume fractions $\left(c_{v}\right)$ $>0.525$, thus are the experiments reported in this paper restricted to $c_{v} \leqslant 0.525$.

Rheological measurements. Stationary flow was established according to the "time-dependent" method (2), viz. by registering the torque experienced by the inner cylinder as a function of time, at one preselected value of the angular velocity of the inner cylinder of the viscometer. Between successive experiments performed on one paste, at different angular velocities, the paste was homogenized by hand. For calculating viscosities only values in the stationary state $(t \rightarrow \infty)$ were employed.

Sedimentation was performed in calibrated glass cylinders of cross section $6.38 \times 10^{-4}$ $\mathrm{m}^{2}$. Both initial sedimentation rate and final sediment volumes were determined at different initial $c_{v}$ values $(0.05 \leqslant c \leqslant 0.15)$. The initial sedimentation rate was measured by following the level at which the turbidity had reached some-arbitrarily chosen-value. From the Stokes formula the "effective sedimenting entity size" was calculated, using the viscosity of the suspension itself and (in the case of aggregation) the density of the final sediment, which was independent of $c_{v}$. The particle sizes thus obtained were extrapolated to $c_{v}=0$ in order to correct for wall friction. The extrapolation was performed linearly from the lowest volume fraction data available. The uncertainties in these values are not such as to endanger the conclusions drawn from them relevant to the present paper. From a comparison with the particle size in those cases where coagulation was absent, we conclude the following about the 
turbidity level chosen as a limit for the sedimenting units. During a sedimentation experiment, first the largest particles leave the volume at the top; at later stages smaller particles follow. At the level assumed, about 80 mass \% of the particles had left the top volume.

\section{RESULTS}

\section{a. Sedimentation}

Table II shows data on hydrodynamic diameters calculated from initial sedimentation rates, extrapolated to $c_{v} \rightarrow 0$, and on solid volume fraction in the final sediment.

The hydrodynamic diameters for hydrophilic particles agree with the values expected from the method of preparation for singlets, and this indicates absence of coagulation in all three media, as had been expected for DOP and glycerol from rheological evidence previously (2). Absence of coagulation is also shown by the final $c_{v}$ values, agreeing with a slightly better than cubical close-packing of equal spheres $\left(c_{v}=0.57\right)$.

Hydrophobic particles, however, show an increasing tendency for coagulation in the direction DOP < DMGP < glycerol. This is again shown both by the initial sedimentation rates and by the final $c_{v}$ values, which are lowered especially in the case of glycerol by formation of a cardhouse structure, on the occurrence of rapid coagulation (5).

Care should be exercised when using the diameters mentioned in Table $I$, in the interpretation of rheological data; for in the latter, shear rates are quite different from those prevailing during the preparation of sedimentation experiments. Nevertheless, the data in Table I indicate a tendency for coagulation in DMGP, and a strong tendency for coagulation in glycerol, of hydrophobic particles. It should be noted, that this direction is that of increasing HLB value of the suspension medium.

\section{b. Rheology}

For suspensions of hydrophilic particles, Newtonian behavior was found up to $c_{v}$ $\approx 0.30$ to 0.35 . The difference between these $c_{v}$ values and those reported previously (2) is ascribed to a more satisfactorily monodisperse character of the particles used in the present work.

At higher $c_{v}$ values, we observed pseudoplatic behavior which could be described, at not too low $\dot{\gamma}$ values, by a Bingham model

$$
\tau=\tau_{\mathrm{B}}+\eta_{\mathrm{PL}} \cdot \dot{\gamma}
$$

Here $\tau_{\mathrm{B}}$ is not a real yield stress, but the constant in the linear $\tau$ vs $\dot{\gamma}$ relation obtained at large $\dot{\gamma}$ values.

The experimental $\tau(\dot{\gamma})$ relations were similar to those reported previously (2). We refrain from using equations like the Mooney (6) or the Dougherty-Krieger (7) relations for estimating, e.g., an effective solid volume fraction for our suspensions, because we consider the theoretical basis of these equations too uncertain, especially in the concentration range where non-Newtonian behavior is found. As a matter of fact, the parameter

TABLE II

Data on Sedimentation

\begin{tabular}{|c|c|c|c|c|c|}
\hline \multirow[b]{3}{*}{ Medium } & \multirow{3}{*}{$\begin{array}{l}\text { HLB } \\
\text { value }\end{array}$} & \multicolumn{4}{|c|}{ Particles $(\mu \mathrm{m})$} \\
\hline & & \multicolumn{2}{|c|}{ Hydrodynamic diameter } & \multicolumn{2}{|c|}{$c_{\mathrm{v}}$ in final sediment } \\
\hline & & Hydrophilic & Hydrophobic & Hydrophilic & Hydrophobic \\
\hline DOP & 4.5 & 36.4 & 36.4 & 0.57 & 0.57 \\
\hline DMGP & 14.6 & 37.2 & 65.0 & 0.57 & 0.54 \\
\hline Glycerol & 20 & 40.0 & 240 & 0.56 & 0.29 \\
\hline
\end{tabular}


$\ln \eta_{\mathrm{r}} /\left(c_{v \max } \cdot \ln \left(1-c_{v} / c_{\max }\right)\right)$ (where $c_{v \max }$ is the maximum solid volume fraction permitting flow), turned out not to be constant for our experiments performed at different $c_{v}$ values, for any reasonable choice of $c_{v \max }$.

Instead, $\tau_{\mathrm{B}}$ and $\eta_{\mathrm{PL}}$ will be plotted separately for the suspensions, as functions of $c_{v}$.

Figure 1 shows $\tau_{\mathrm{B}}$ as a function of $c_{v}$. Although $\tau_{\mathrm{B}}$ starts to deviate from zero at about the same $c_{v}$ value for the three media, its increase with increasing $c_{v}$ at higher $c_{v}$ values is not equal in the three media: $d \tau_{\mathrm{B}} /$ $d c_{v}$ increases in the direction DMGP $<$ DOP $<$ glycerol, thus in the direction of increasing viscosity of the suspension medium $\left(\eta_{0}\right)$.

Suspensions of hydrophobic particles were characterized by pseudoplastic behavior at decreasing $c_{v}$ values, in the direction of increasing HLB value of the medium (Fig. 2). The $d \tau_{\mathrm{b}} / d c_{v}$ at larger $c_{v}$ values than those corresponding with the onset of deviations from Newtonian behavior, however, is not connected with the HLB value of the medium but again with $\eta_{0}$. In fact, $d \tau_{\mathrm{B}} / d c_{v}$ at large $c_{v}$ values is in each medium approximately equal for suspensions of hydrophilic and hydrophobic particles (cf. Figs. 1 and 2).

Values of $\eta / \eta_{0}$ for Newtonian systems and of $\eta_{\mathrm{PL}} / \eta_{0}$ for pseudoplastic ones are plotted against $c_{v}$ in the Figs. 3 and 4 . For suspensions

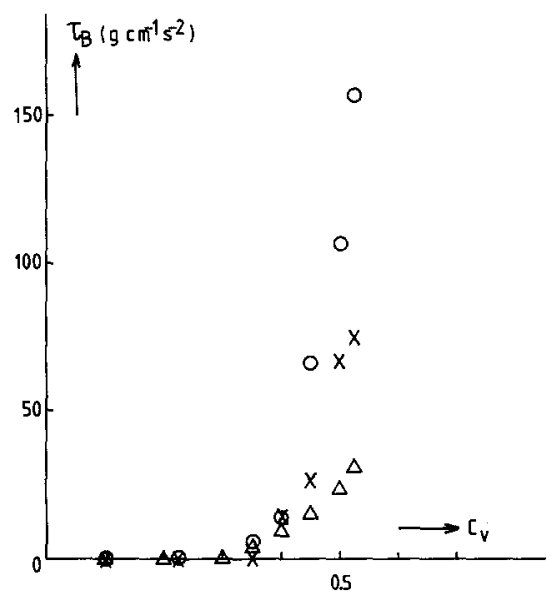

FIG. 1. $\tau_{\mathrm{B}}$ vs $c_{v}$ for suspensions of hydrophilic particles. $(\times)$ DOP, $(\triangle)$ DMGP, (O) glycerol.

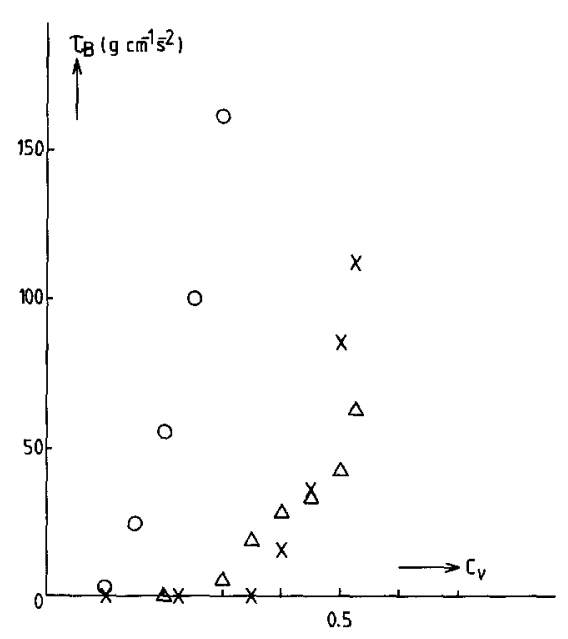

FIG. 2. $\tau_{\mathrm{B}}$ vs $c_{\mathrm{v}}$ for suspensions of hydrophobic particles. $(\times)$ DOP, $(\triangle)$ DMGP, $(O)$ glycerol.

of hydrophilic particles the curves coincide within experimental error, but in the case of hydrophobic particles a strong tendency to coagulation (as in the case of suspensions in

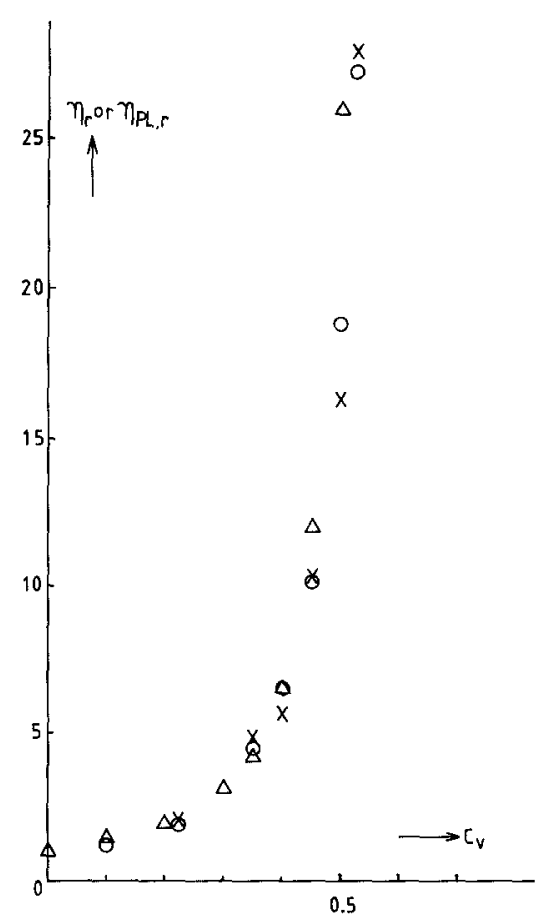

FIG. 3. $\eta / \eta_{0}$ or $\eta_{\mathrm{PL}} / \eta_{0}$ vs $c_{v}$, for suspensions of hydrophilic particles. $(\times)$ DOP, $(\triangle)$ DMGP, $(O)$ glycerol. 


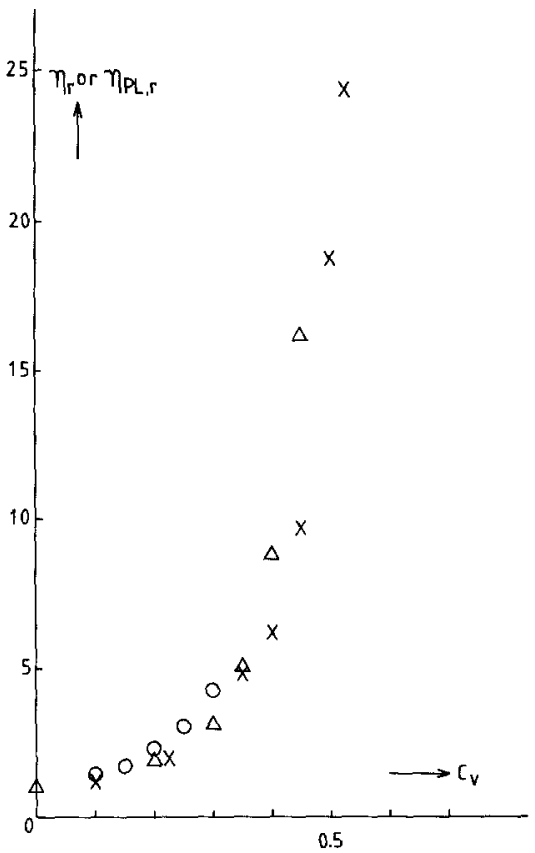

FIG. 4. $\eta / \eta_{0}$ or $\eta_{\mathrm{PL}} / \eta_{0}$ vs $c_{v}$, for suspensions of hydrophobic particles. $(\times)$ DOP, $(\triangle)$ DMGP, $(O)$ glycerol.

glycerol) is shown by a slightly larger $\eta_{\mathrm{PL}} / \eta_{0}$ value than found for suspensions with no coagulation, at the same $c_{v}$ values.

It follows that suspensions of hydrophilic and hydrophobic particles in DOP show within experimental error the same rheological behavior.

\section{DISCUSSION}

Aggregate formation appears to be negligible in suspensions of hydrophilic particles in the three media investigated, but for suspensions of hydrophobic particles it becomes progressively more important from DOP (where it is negligible) through DMGP to glycerol. In view of the rather weak (though distinct) tendency toward coagulation in DMGP, a HLB value of about 14 appears to be the limit at which coagulation of hydrophobic values becomes important.

In glycerol, on the other hand, the coagulation tendency of hydrophobic particles is much stronger. The slight increase in $\eta_{\mathrm{PL}} / \eta_{0}$ values above those obtained for suspensions in other media at the same $c_{v}$ values (Fig. 4) indicates a fargoing but not complete breakdown of coagulation structure in the $\dot{\gamma}$ region in which a linear $\tau$ vs $\dot{\gamma}$ curve is approached $\left(\dot{\gamma} \approx 100 \mathrm{sec}^{-1}\right)$.

This means that some bonds between particles at least must be able to withstand the largest shearing stress exerted upon a couple by the surroundings at those $\dot{\gamma}$ values.

The maximum frictional force experienced by a member of a pair in the direction of the line joining their centers, will be

$$
F_{\text {fr }}=6 \pi \eta_{0} f b^{2} \dot{\gamma}
$$

with $f=$ the frictional coefficient.

$f$ will be about 1 : the vicinity of a neighboring particle decreases $f$ slightly (8); on the other hand, the presence of other particles around the pair considered increases $f$ but not to a large extent, because direct collisions with these other particles will be avoided. Thus the maximum value of $F_{\mathrm{fr}}$ in glycerol can be calculated, for $b=40 \mu \mathrm{m}$ and $\dot{\gamma}$ $=100 \mathrm{sec}^{-1}$, to be about $5 \times 10^{-7} \mathrm{~N}$.

In order to compare this with other values for attractive forces between spherical particles, we start from the attractive potential energy at short separation, as given by the Hamaker equation (9).

The attractive force between the two spheres at short separations $S_{0}$ of the surfaces becomes

$$
\frac{d V_{\mathrm{A}}}{d S_{0}}=\frac{A b}{12 S_{0}^{2}}
$$

with $A=$ the Hamaker constant.

By estimating $S_{0}$ and comparing $d V_{\mathrm{A}} / d S_{0}$ with the maximum value for $F_{\mathrm{fr}}$, a value for the Hamaker constant can be evaluated. For $S_{0}$, values between 0.2 and $0.4 \mathrm{~nm}$ have been reported (10-12). A value of $0.3 \mathrm{~nm}$ would lead to a Hamaker constant of $0.6 \times 10^{-20} \mathrm{~J}$ which compares well with the values reported for vitreous quartz in water $(13,14)$. It should be remembered that the $S_{0}$ value employed is likely to be underestimated, in view of deviations from the exact spherical shape expected for the glass Ballotini. Thus, 
the value for the Hamaker constant will be an underestimate as well. Indeed, for hydrophobic glass particles in water a larger value of $A$ is expected than the values reported for hydrophilic vitreous quartz fibers in water, reported by Deryagin, Rabinovich, and Churaev $(13,14)$.

It should be remarked that deviations from the exact spherical shape may lead to a larger attraction, than shown by spheres by permitting more than just one contact point. This may, at least for part of the contacts between the particles, outweigh the effect of the more restricted character of a contact point between nonspherical particles. At any rate, much larger $S_{0}$ values would lead to unrealistic values of the Hamaker constant.

The values of $d \tau_{B} / d c_{v}$ at $c_{v}$ values corresponding with pseudoplastic behavior are illuminating with regard to the question: which processes contribute significantly to the energy dissipation during stationary flow (2)?

It can be thought to be composed of two main parts (15-19):

1. That occurring in a suspension of the same effective $c_{v}$ and aggregate (or: particle) size distribution, but involving only hydrodynamic interaction between the aggregates (particles);

2. That due to motion of particles within the aggregates. It should be noted that in the absence of coagulation, at large $c_{v}$ values loose aggregates such as layers may arise through the ordering effect of shear $(2,20)$. The ordering through shear observed at large $c_{v}$ values $(21)$ is consistent with this formation of loose aggregates.

The energy dissipation part 2 may in turn be divided into: a. energy dissipation due to increase and decrease of interparticle potential energy (i.e., stretching or breaking and renewed formation of bonds between them); b. energy dissipation connected with the overcoming of viscous drag experienced by the particles in two colliding flocs (or layers gliding over each other).
Among these effects, that labeled $2 a$ differs from the other effects because, at a given displacement of the particles from their equilibrium position, the energy dissipation is not proportional to $\eta_{0}$. The interparticle potential energy may depend on the nature of the medium through variation of the Hamaker constant, but a direct proportionality of the effects with the viscosity of the suspension medium would be fortuitous.

Earlier results had led some authors (2, 15-19) to the conclusion that breaking and renewed formation of interparticle bonds does not contribute significantly to $\epsilon$. However, the reverse has been argued as well $(22,23)$.

From an experimental point of view, the energy dissipation per unit of volume and time can be calculated for simple shear, from (24)

$$
\epsilon=\tau \cdot \dot{\gamma}
$$

which for a Bingham liquid becomes

$$
\epsilon=\tau_{\mathrm{B}} \cdot \dot{\gamma}+\eta_{\mathrm{PL}} \cdot \dot{\gamma}^{2}
$$

Now the coincidence of the curves $\eta / \eta_{0}$ and $\eta_{\mathrm{PL}} / \eta_{0}$ in the case of hydrophilic particles (Fig. 3), and the near coincidence of these curves in the case of hydrophobic particles, suggest that $\eta$ and $\eta_{\mathrm{PL}}$ are proportional to $\eta_{0}$, the viscosity of the suspension medium. Sim-

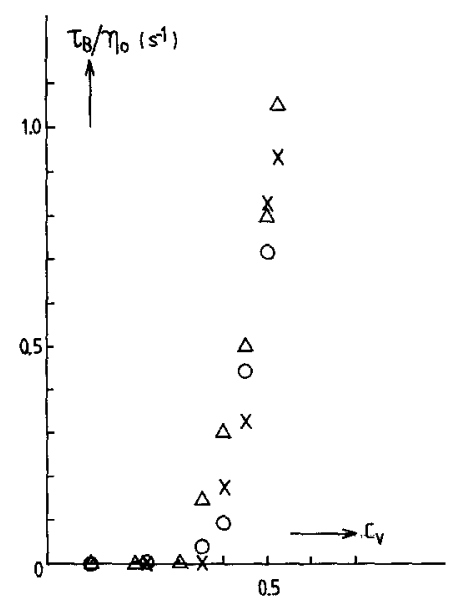

FIG. 5. $\tau_{\mathbf{B}} / \eta_{0}$ vs $c_{v}$ for suspensions of hydrophilic particles. $(\times)$ DOP, $(\triangle)$ DMGP, $(O)$ glycerol. 
ilarly, the fact that $d \tau_{\mathrm{B}} / d c_{v}$ in pseudoplastic fluids increases with increasing $\eta_{0}$, suggests that $\tau_{\mathrm{B}}$ is proportional to $\eta_{0}$. Indeed, if $\tau_{\mathrm{B}} /$ $\eta_{0}$ is plotted vs $\mathcal{C}_{v}$, the curves are found to coincide within experimental error for suspensions of hydrophilic particles (Fig. 5) and to become disentangled for hydrophobic ones (Fig. 6).

On the other hand, there appears to be no direct proportionality between $\tau_{B}$ and interparticle potential energy: the latter is larger in absolute sense in DMGP than in DOP (cf. the HLB values and the sedimentation data), yet at $c_{v}>0.45 \tau_{\mathrm{B}}$ in DOP is larger than in DMGP.

These facts are consistent with the hypothesis that both $\tau_{\mathrm{B}}$ and $\eta_{\mathrm{PL}}$ are proportional to $\eta_{0}$. This means, in view of Eq. [5], that $\epsilon$ is proportional to $\eta_{0}$ (at given $c_{v}$ and $\dot{\gamma}$ values). Thus, increase and decrease in interparticle potential energy does not contribute significantly to the energy dissipation during stationary flow, and the latter is determined predominantly by the energy needed for overcoming the viscous drag experienced either by aggregates or by particles moving

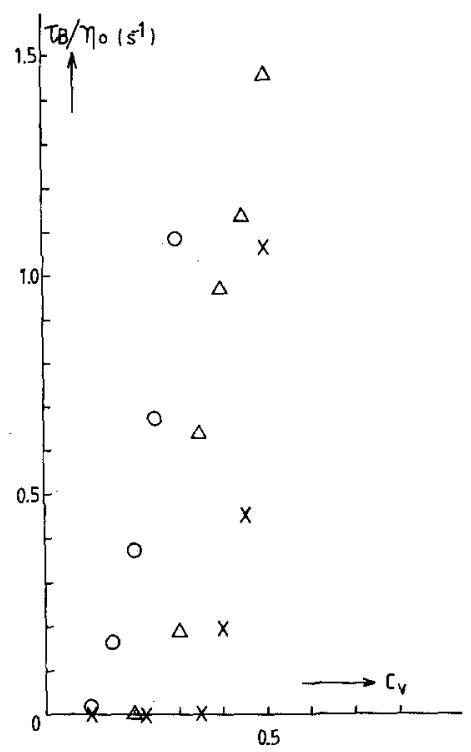

FIG. 6, $\tau_{\mathrm{B}} / \eta_{0}$ vs $c_{v}$ for suspensions of hydrophobic particles. $(\times)$ DOP, $(\triangle)$ DMGP, $(O)$ glycerol. within aggregates. Coagulation affects this viscous drag by increasing the frictional coefficient: particles in aggregates have, because of the nearby presence of other particles, a larger frictional coefficient than singlets.

It should be noted that this conclusion is based on experimental data obtained for suspensions of relatively large particles, and should not without further evidence be extended to dispersions of much smaller particles.

\section{CONCLUSIONS}

1. Hydrophilic glass particles do not noticeably coagulate in organic media with HLB $>4.5$.

2. Hydrophobic glass particles coagulate if HLB $>14$.

3. There is no significant contribution to energy dissipation during stationary flow, from increase and decrease of interparticle potential energy (breaking and renewed formation of bonds).

APPENDIX: NOMENCLATURE

A Hamaker constant

$b \quad$ radius of a primary particle

$c_{v} \quad$ solid volume fraction

DMGP phthalic acid bis-methyl glycol ester DOP di-2-ethylhexyl phthalate

$d \quad$ hydrodynamic diameter

$F_{\mathrm{fr}} \quad$ maximum frictional force experienced by a member of a pair of contacting spheres, in the direction of the line joining the centers of two contacting spheres

$f \quad$ frictional coefficient

HLB hydrophilic/lipophilic balance

$S_{0} \quad$ separation of surfaces between two contacting particles

$V_{\mathrm{A}} \quad$ attractive interparticle potential energy

$\gamma \quad$ shear rate

$\epsilon \quad$ energy dissipation per unit of volume and time viscosity viscosity of the suspension medium 
$\eta_{\mathrm{PL}} \quad$ slope in linear $\tau$ vs $\dot{\gamma}$ relation

$\tau$ shear stress

$\tau_{\text {B }} \quad$ constant in linear $\tau$ vs $\dot{\gamma}$ relation

$\bar{\eta}_{\mathrm{r}} \quad$ relative viscosity $=\eta / \eta_{0}$

\section{REFERENCES}

1. Kao, S. V., Nielsen, L. E., and Hill, C. T., J. Colloid Interface Sci. 53, 358, 367, (1975).

2. van Diemen, A. J. G., and Stein, H. N., J. Colloid Interface Sci. 86, 318 (1982).

3. Fordyce, C. R., and Meyer, L. W. A., Ind. Eng. Chem. 32, 1054 (1940).

4. "Kirk-Othmer's Encyclopedia of Chemical Technology," Third ed., Vol. 8, pp. 910-918. Wiley, New York, 1979.

5. Hiemenz, P. C., "Principles of Colloid and Surface Chemistry," p. 24. Dekker, New York, 1977.

6. Mooney, M., J. Colloid Sci. 6, 162 (1951).

7. Krieger, J. M., Adv. Colloid Interface Sci. 3, 111 (1972).

8. Gluckman, M. J., Pfeffer, R., and Weinbaum, S., J. Fluid Mech. 50, 705 (1971).

9. Hiemenz, P. C., "Principles of Colloid and Surface Chemistry," p. 414. Dekker, New York, 1977.

10. Krupp, H., Adv. Colloid Interface Sci. 1, 111 (1967).
11. Neumann, A. W., Omenyi, S. N., and van Oss, C. J., Colloid Polym. Sci. 256, 413 (1979).

12. Visser, J., Adv. Colloid Interface Sci. 15, 157 (1981).

13. Deryagin, B. V., Rabinovich, Y. I., and Churaev, N. V., Nature (London) 265, 520 (1977).

14. Deryagin, B. V., Rabinovich, Y. I., and Chruaev, N. V., Nature (London) 272, 313 (1978).

15. Firth, B. A., and Hunter, R. J., J. Colloid Interface Sci. 57, 248, 266 (1976).

16. Firth, B. A., J. Colloid Interface Sci. 57, 257 (1976).

17. van de Ven, T. G. M., and Hunter, R. J., Rheol. Acta 16, 534 (1977).

18. van Diemen, A. J. G., and Stein, H. N., Rheol. Acta 22, 41 (1983).

19. van Diemen, A. J. G., and Stein, H. N., in "The Role of Particle Interactions in Powder Mechanics" (Th. M. G. Senden and A. H. M. Verkooijen, Eds.), p. 261. preprints of the International Symposium, Eindhoven, August 29-31, 1983.

20. Hoffman, R. L., Adv. Colloid Interface Sci. 17, 161 (1982).

21. Russel, W. B., J. Rheol. (N, Y.) 24, 287 (1980).

22. Luckham, P. F., Vincent, B., and Tadros, Th. F., Colloids Surf. 6, 101 (1983).

23. Heath, D., and Tadros, Th. F., Discuss. Faraday Soc. 76, (1983) (quoted from preprint).

24. Bird, R. B., Stewart, W. E., and Lightfoot, E. N., "Transport Phenomena," p. 314. Wiley, New York, 1960. 